\title{
Controle da síntese de prostaglandina F2 $\alpha$ no endométrio bovino in vitro
}

Claudia Maria BERTAN ${ }^{1}$

Pauline Martins da CUNHA ${ }^{1}$

Claudio Alvarenga de OLIVEIRA $^{1}$

Anneliese de Souza TRALDI ${ }^{1}$ Mario BINELLI ${ }^{1}$

\section{Correspondência para:}

CLÁUDIA MARIA BERTANI

Centro de Biotecnologia em Reprodução

Animal

Departamento de Reprodução Animal

Faculdade de Medicina Veterinária e

Zootecnia

Universidade de São Paulo

Av. Duque de Caxias Norte, 225

13630-000 - Pirassununga - SP

binelli@usp.br

Recebido para publicação: 06/07/2004 Aprovado para publicação: 01/06/2005

1 - Departamento de Reprodução Animal da Faculdade de Medicina Veterinária e Zootecnia da Universidade de São Paulo, Pirassununga - SP

\section{Resumo}

Palavras-chave: Bovinos. Prostaglandina.

A síntese de prostaglandina F2 $\alpha$ (PGF2 $\alpha$ ) endometrial, resulta de uma complexa cascata de eventos intracelulares que ocorrem de maneira altamente coordenada. A síntese de PGF2 $\alpha$ pode ser estimulada na presença da melitina e do forbol 12,13 dibutirato (PDBu). O objetivo do presente estudo foi verificar se a produção basal e a estimulação aguda de PGF2 $\alpha$ são dependentes da síntese de proteínas. No experimento 1, explantes obtidos de fêmeas bovinas $(n=2)$, cíclicas, não lactantes, no dia 15 do ciclo estral foram incubados em quadruplicata, com meio de cultivo (KHB) ou KHB suplementado com PDBu $10^{-6} \mathrm{M}$, melitina $10^{-6} \mathrm{M}$ ou melitina $10^{-5} \mathrm{M}$. Amostras do meio foram coletadas 0 e 60 minutos após os tratamentos e as concentrações de PGF2 $\alpha$ foram mensuradas por radioimunoensaio. Com 60 minutos após os tratamentos houve aumento das concentrações médias de PGF2 $\alpha(\mathrm{P}<0,06)$ em resposta ao tratamento com $\mathrm{PDBu}$ comparado ao grupo KHB e melitina. No experimento 2 , explantes endometriais de fêmeas bovinas ( $n=4)$, não gestantes, no $17^{\circ}$ dia do ciclo estral, pesando de 80 a $100 \mathrm{mg}$ foram incubados em KHB suplementado com 0, 50, 100 ou $200 \mathrm{mg} / \mathrm{mL}$ de CHX e 0 ou $100 \mathrm{ng} / \mathrm{mL}$ de PDBu em um arranjo fatorial $2 \mathrm{x} 4$, em quadruplicata. Amostras do meio foram coletadas 0 e 60 minutos após os tratamentos e as concentrações de PGF2 $\alpha$ foram mensuradas por radioimunoensaio. A integridade dos explantes endometriais tratados com CHX foi avaliada por cortes histológicos. Foi observado aumento na produção de PGF2 $\alpha$ em resposta ao tratamento com PDBu $(\mathrm{P}<0,01)$, entretanto, não houve diferenças na produção de PGF2 $\alpha$ pelos tecidos tratados com diferentes concentrações de $\mathrm{CHX}$, associadas ou não ao PDBu. A integridade histológica dos explantes foi preservada. Conclui-se que a produção basal e a estimulação aguda da síntese de PGF2 $\alpha$ não são dependentes da síntese de novas proteínas.

\section{Introdução}

É de amplo conhecimento que a prostaglandina F2 $\alpha$ (PGF2 $\alpha$ ), sintetizada principalmente pelas células epiteliais da região intercaruncular do endométrio ${ }^{1,2,3}$ é o principal agente luteolítico em fêmeas bovinas. A PGF $2 \alpha$ tem como função bloquear a síntese de progesterona no corpo lúteo ${ }^{4,5,6,7}$ determinando o final da fase luteínica e do ciclo estral ${ }^{9,10}$. Em fêmeas bovinas, a luteólise ocorre normalmente entre os dias $15^{\circ}$ e $19^{\circ}$ do ciclo estral. Em um intervalo de 48 a 72 horas, cinco a oito pulsos de PGF2 $\alpha$ são liberados pelo endométrio e promovem a regressão do corpo lúteo ${ }^{11,12,13,14,15}$.

A síntese de PGF2 $\alpha$ nas células endometriais resulta de uma complexa cascata de eventos intracelulares que ocorrem de maneira altamente coordenada. Essa cascata envolve a ativação seqüencial da proteína ligadora da guanosina trifosfato (proteína G) que promove a ativação da fosfolipase C (PKC) ${ }^{16}$. A PLC estimula a conversão do fosfatidil inositol bifosfato em 
inositol trifosfato $\left(\mathrm{IP}_{3}\right)$ e diacilglicerol. $\mathrm{O} \mathrm{IP}_{3}$ estimula a liberação de cálcio pelo retículo endoplasmático promovendo um aumento das concentrações de cálcio no citoplasma, estímulo que ativa a PKC. Por outras vias o diacilglicerol também estimula a atividade da $\mathrm{PKC}^{17}$. A PKC estimula a fosfolipase $\mathrm{A}_{2}$ $\left(\mathrm{PLA}_{2}\right)^{18}$ que promove a liberação de ácido araquidônico da membrana fosfolipídica para a célula ${ }^{19}$. O ácido araquidônico pela ação da ciclooxigenase $2{ }^{20}$ é convertido em prostaglandina $\mathrm{H}_{2}\left(\mathrm{PGH}_{2}\right)$. Por fim, a enzima prostaglandina sintase converte a $\mathrm{PGH}_{2} \mathrm{em}$ PGF2 $\alpha^{21}$.

A síntese de PGF2 $\alpha$ em explantes endometriais pode ser estimulada pela melitina, que estimula a atividade da PLA 16,22,23,24 e do forbol 12,13 dibutirato (PDBu) um análogo do diacilglicerol que estimula $\mathrm{PKC}^{25,26}$.

O objetivo do presente estudo foi verificar se as produções basal e estimulada de PGF2 $\alpha$ são dependentes da síntese de proteínas.

\section{Materiais e Métodos}

\section{Local do experimento e animais}

$O$ experimento foi realizado na Universidade de São Paulo, na Faculdade de Medicina Veterinária e Zootecnia, no Centro de Biotecnologia em Reprodução Animal, no Laboratório de Fisiologia e Endocrinologia Molecular, no Campus de Pirassununga - SP. Foram utilizados explantes endometriais de fêmeas bovinas mestiças (Bos taurus taurus $\mathrm{x}$ Bos taurus indicus), não gestantes, no $15^{\circ}(\mathrm{n}=2$; experimento 1) ou no $17^{\circ} \quad(\mathrm{n}=4$; experimento 2) dia do ciclo estral. Os estros foram sincronizados com uma injeção intramuscular de PGF2 $\alpha 150 \mathrm{mg}$ de Dcloprostenol (N.C.Preloban ${ }^{\circledR}$ - Intervet).

\section{Delineamentos experimentais.}

\section{Experimento 1}

Imediatamente após o abate, o sistema genital foi transportado ao laboratório a $4^{\circ} \mathrm{C}$. O endométrio do corno uterino ipsolateral ao corpo lúteo (CL) foi dissecado e fragmentos da região intercaruncular de 80 a $100 \mathrm{mg}$ foram acondicionados em tubos de borosilicato 12 x $75 \mathrm{~mm}$ contendo $0,5 \mathrm{~mL}$ de meio bicarbonato Krebs-Hensleit (KHB; $118 \mathrm{mM}$ $\mathrm{NaCl}, 4.7 \mathrm{mM} \mathrm{KCl}, 2.56 \mathrm{mM} \mathrm{CaCl}, 1.13$ $\mathrm{mM} \mathrm{MgCl}, 25 \mathrm{mM} \mathrm{NaHCO}, 1.15 \mathrm{mM}$ $\mathrm{NaH}_{2} \mathrm{PO}_{4}, 5.55 \mathrm{mM}$ glucose, $20 \mathrm{mM}$ Hepes, e $0.013 \mathrm{mM}$ de vermelho fenol; $\mathrm{pH}$ 7.4). O cultivo dos explantes endometriais foi realizado conforme a técnica descrita por Burns et al. ${ }^{16}$. Os tubos foram colocados em banho-maria a $37^{\circ} \mathrm{C}$ com agitação (40rpm) durante 1 hora. A seguir, descartou-se o meio de cultura e lavaram-se os explantes duas vezes com $\mathrm{KHB}$ e adicionou-se $0,5 \mathrm{~mL}$ de KHB por tubo. Após nova incubação por uma hora, repetiu-se a lavagem. Os fragmentos endometriais de cada animal foram incubados em $1 \mathrm{~mL}$ dos seguintes tratamentos em quadruplicata: KHB (controle) ou KHB suplementado com Melitina $10^{-6} \mathrm{M}$, Melitina $10^{-5} \mathrm{M}$ ou PDBu $10^{-}$ ${ }^{6} \mathrm{M}$. Amostras de $100 \mathrm{~mL}$ do meio de cultura foram removidas imediatamente e 60 minutos após a adição dos tratamentos e armazenadas a $-20^{\circ} \mathrm{C}$ para a mensuração das concentrações de PGF $2 \alpha$ por radioimunoensaio ${ }^{27}$.

Análise Estatística

Demonstrou-se com uma análise preliminar que os dados não estavam adequados quanto à normalidade dos resíduos (Teste Shapiro-Wilk, $\mathrm{P} \leq 0,01$ ) e homogeneidade das variâncias (Teste $\mathrm{F}$, $\mathrm{P} \leq 0,01)$. Os dados foram então transformados pelo método Box-Cox $(\text { lambda }=0.6)^{28}$. Tal transformação adequou os dados à análise de variância (Shapiro-Wilk e Teste F; $\mathrm{P} \geq 0,01$ ) os quais estão apresentados como LSmeans \pm EPM, não transformados. A concentração de PGF $2 \alpha$ em $\mathrm{pg} / \mathrm{mL} / \mathrm{mg}$ de tecido endometrial foi analisada por ANOVA, utilizando-se o proc GLM do programa SAS (SAS Institute ${ }^{28}$ ). As variáveis independentes foram: animal, tratamento e 
tratamento $\mathrm{x}$ animal. As médias dos tratamentos foram separadas por contrastes ortogonais.

\section{Experimento 2}

O procedimento de cultivo e lavagens dos explantes endometriais foi realizado da mesma maneira que no experimento 1. Os fragmentos endometrais de cada animal foram incubados em $1 \mathrm{~mL}$ de $\mathrm{KHB}$ suplementado com $0,50,100$ ou $200 \mu \mathrm{g} / \mathrm{mL}$ de CHX e 0 ou $100 \mathrm{ng} / \mathrm{mL}$ de PDBu $\left(10^{-5} \mathrm{M}\right)$ em um arranjo fatorial 4 x 2 , em quadruplicata. Amostras de $100 \mu \mathrm{L}$ do meio de cultura foram removidas imediatamente e 60 minutos após a adição dos tratamentos e armazenadas a $-20^{\circ} \mathrm{C}$ para a mensuração das concentrações de PGF2 $\alpha$, por radioimunoensaio. Ao final do período de incubação, amostras de tecido endometrial de todos os tratamentos foram coletadas, fixadas em formol e incluídas em parafina. Cortes histológicos foram preparados e corados por hematoxilina-eosina para a avaliação da integridade dos tecidos por microscopia óptica.

\section{Análise Estatística}

Demonstrou-se com uma análise preliminar que os dados não estavam adequados quanto à normalidade dos resíduos (Teste Shapiro-Will, $\mathrm{P} \leq 0,01)$ e homogeneidade das variâncias pelo (Teste $\mathrm{F}, \mathrm{P} \leq 0,01$ ). Os dados foram então transformados por raiz quadrada. Tal transformação adequou os dados à análise de variância (Shapiro-Wilk e Teste F; P $\geq 0,01$ ) os quais estão apresentados como LSmeans \pm EPM, não transformados. A produção de PGF $2 \alpha \mathrm{em} \mathrm{pg} / \mathrm{mL} / \mathrm{mg}$ de tecido aos 60 minutos foi analisada por ANOVA, utilizandose o proc GLM do programa SAS (SAS Institute $\left.^{28}\right)$. As variáveis independentes foram: animal, tratamento e a interação tratamento $x$ animal. As médias foram separadas por contrastes ortogonais.

\section{Resultados e Discussão}

\section{Experimento 1}

Houve interação tratamento $\mathrm{x}$ animal $(\mathrm{P}<0,05)$ nas concentrações médias de PGF2 $\alpha$ 60 minutos após a adição dos diferentes tratamentos. As médias dos tratamentos na fêmea utilizada na primeira repetição foram proporcionalmente menores que na segunda. Foi observada uma tendência da produção de PGF2 $\alpha$ ter sido maior nos explantes tratados com $\mathrm{PDBu}(\mathrm{P}<0,06$; Figura1) quando comparados ao demais tratamentos. A produção de PGF2 $\alpha$ estimulada pelo PDBu foi 94\% maior que para o controle.

A síntese de PGF2 $\alpha$, estimulada pelo PDBu em explantes endometriais foi observada em outros experimentos ${ }^{29,30,31}$. Arnold et al. ${ }^{29}$ verificaram em explantes obtidos de vacas no $17^{\circ}$ dia do ciclo estral, que o PDBu $10^{-5} \mathrm{M}$ estimulou uma maior secreção de PGF2 $\alpha$ comparado ao grupo controle. As concentrações médias de PGF2 $\alpha$, 60 minutos após os tratamentos, foram de 50,82 e $87,77 \mathrm{pg} / \mathrm{mL} /$ mg de tecido para os explantes endometriais controle e tratados com $\mathrm{PDBu}$, respectivamente, semelhante ao presente estudo.

A capacidade responsiva do endométrio ao $\mathrm{PDBu}$ foi verificada no $15^{\circ}$ e $17^{\circ}$ dias do ciclo estral. A presença do PDBu no meio de cultivo foi suficiente para acionar a maquinaria enzimática necessária para a síntese de PGF2 $\alpha$ através da ativação da PKC, demonstrando que os demais componentes da cascata geradora de PGF2 $\alpha$, subseqüentes a PKC, estavam presentes nas células endometriais e em concentrações suficientes, proporcionando uma resposta estimulatória bastante rápida, 60 minutos após o tratamento.

Em relação ao controle não houve diferença na produção de PGF2 $\alpha$ por explantes tratados com melitina nas doses utilizadas (Figura1). Tanto Burns et al. ${ }^{16}$ como Arnold et $\mathrm{al}^{2}$ reportaram estímulo à produção de PGF2 $\alpha$ com melitina, nas concentrações de $10^{-6} \mathrm{M}$ e 10 ${ }^{5} \mathrm{M}$, respectiva-mente.

A melitina estimula a síntese de PGF2 $\alpha$ no $17^{\circ}$ dia do ciclo estral ${ }^{16,29}$ e não no $15^{\circ}$ dia do ciclo, conforme observado do presente estudo. É possível que haja diferenças no preparo da maquinaria enzimática para a estimulação da síntese de PGF2 $\alpha$ pela melitina entre os dias $15 \mathrm{e} 17$ do ciclo estral. Possivelmente, no $15^{\circ}$ dia o estímulo da atividade da $\mathrm{PLA}_{2}$ nas células endometriais pela melitina não são 
suficientes para acionar a cascata geradora de PGF2 $\alpha$.

\section{Experimento 2}

Foi observado efeito de tratamento, havendo um aumento na produção de PGF2 $\alpha$ nos tecidos tratados com $\mathrm{PDBu}(\mathrm{P}<0,01$, Figura 2).

A produção de PGF2 $\alpha$ basal ou induzida pelo PDBu não foi sensível ao tratamento com CHX nas concentrações utilizadas. É possível que tais concentrações de CHX não tenham sido suficientes para alterar a síntese de proteínas envolvidas na produção de PGF2 $\alpha$. Contudo, reportou-se efetiva a inibição da síntese de proteínas quando foram utilizadas concentrações de CHX de $0,1 \mathrm{a} 10 \mu \mathrm{g} / \mathrm{mL}^{1}$. Portanto, parece improvável que as concentrações de CHX utilizadas no presente estudo tenham sido insuficientes para alterar a síntese de proteínas. Alternativamente, sugere-se que a síntese de PGF2 $\alpha$ estimulada pelo PDBu seja independente da síntese de proteínas. Considerando que houve uma resposta estimulatória ao PDBu já aos 15 minutos após o tratamento (dados não apresentados) épossivel que a maquinaria enzimática localizada a jusante da PKC estava pronta, e bastou estimular esta proteína quinase para que a produção de PGF2 $\alpha$ fosse estimulada. Tal estímulo pelo $\mathrm{PDBu}$ foi consistente ao descrito para o experimento 1 do presente trabalho.

Especulou-se que concentrações elevadas da CHX causariam injúrias ao tecido endometrial, uma vez que se trata de um inibidor da síntese de proteínas. Os exames histopatológicos realizados nos fragmentos de endométrio, demonstraram que a integridade do tecido em questão foi preservada após a incubação. Ao exame histopatológico não foi revelada nenhuma diferença estrutural em resposta às diferentes concentrações de $\mathrm{CHX}$ (dados não apresentados). Uma vez que não foram detectados efeitos de toxicidade da CHX nos explantes, entendeu-se que a concentração de $200 \mu \mathrm{g} / \mathrm{mL}$ de CHX, a dose mais elevada nesse estudo, seria a mais adequada para experimentos futuros com cultivos de endométrio de maior duração.

\section{Conclusão}

As produções basal e estimulada de

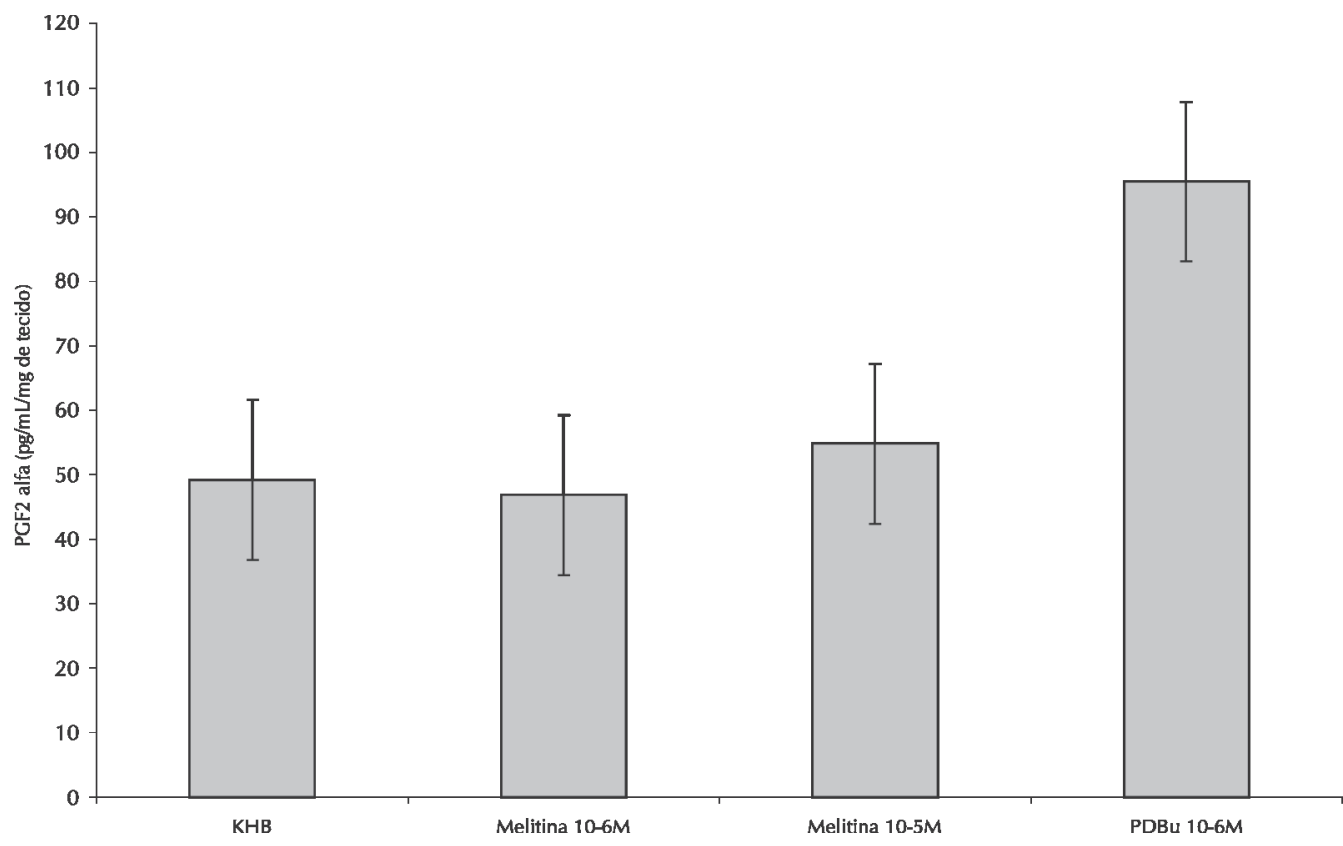

Figura 1 - Experimento 1 : Médias das concentrações de PGF2a produzidas por explantes endometriais de animais, no $15^{\circ}$ dia do ciclo estral, 60 minutos após a administração de KHB (controle), Melitina $10^{-6} \mathrm{M}$, Melitina $10^{-5} \mathrm{M}$ ou PDBu $10^{-6} \mathrm{M}$ (valores não transformados; LSmeans + EPM). Médias com sobrescritos diferentes diferiram estatisticamente $(P<0,06)$ 


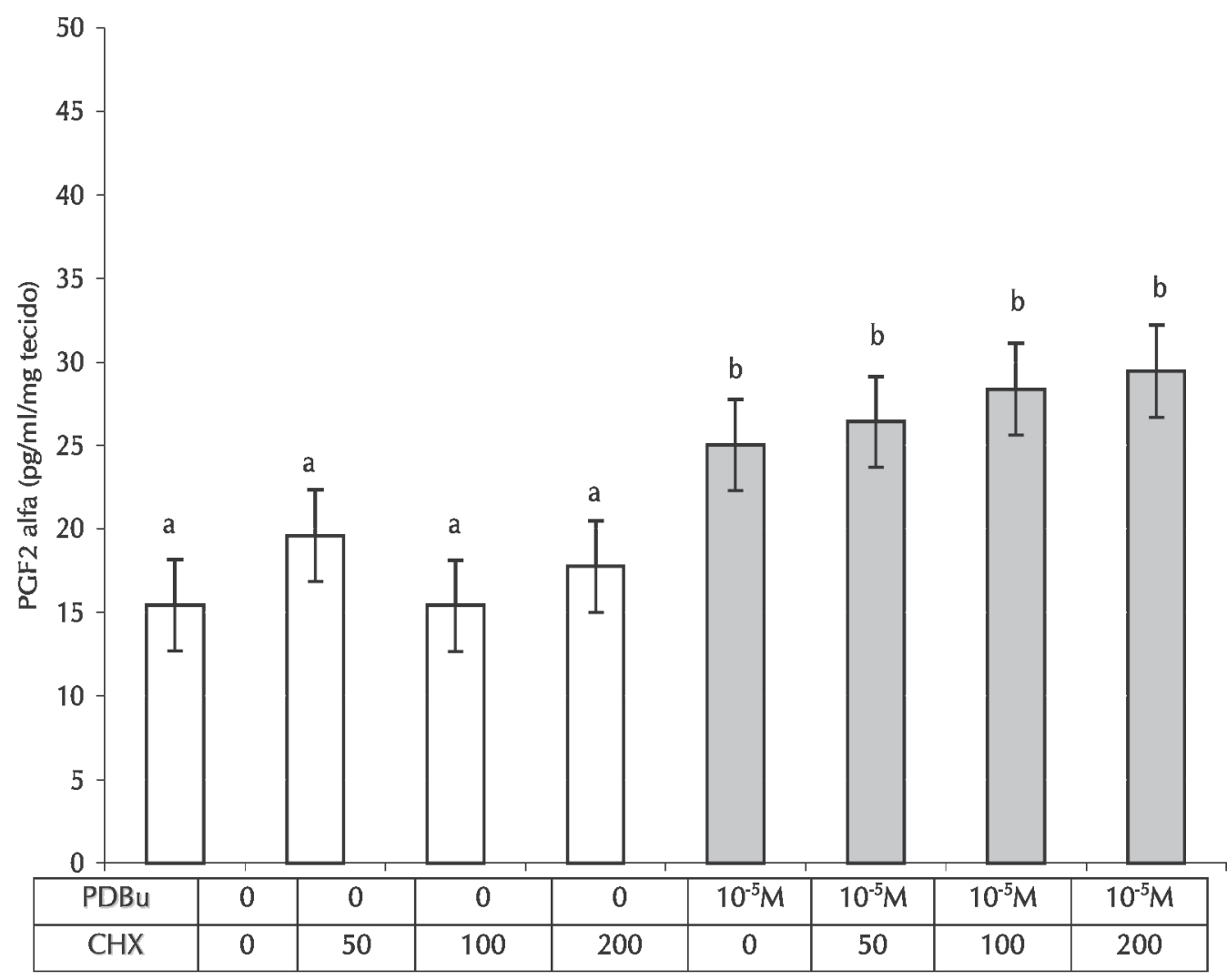

Figura 2 - Experimento 2 : Médias das concentrações de PGF2a produzidas por explantes de endométrio de animais no $17^{\circ}$ dia do ciclo estral, 60 minutos após a administração de $0,50,100$ ou 200mg/mL CHX combinados com 0 ou 100 $\mathrm{mg} / \mathrm{mL}$ PDBu (valores não transformados; LSmeans + EPM). Médias com sobrescritos diferentes diferiram estatisticamente $(P<0,01)$

PGF2 $\alpha$ não são dependentes da síntese de proteínas, ou seja, a maquinaria enzimática necessária para a síntese de PGF2 $\alpha$ encontrase presente, funcional e responsível a estímulos agudos, como o fornecido pelo PDBu.

\section{Instituição de patrocínio}

Fundação de Amparo à Pesquisa do Estado de São Paulo - FAPESP: Processos 00/07117-0 de CMB; 01/12442-0 de PMC e 99/03383-9 de MB.

\section{Agradecimentos}

Ao Prof. Dr. William Thatcher, da Universidade da Flórida, pela doação das células BEND e dos anticorpos de PGF2 $\alpha$ utilizados nas técnicas de radioimunoensaio.

A Profa. Dra. Maria Angélica Miglino, a Profa. Dra. Paula de Carvalho Papa e a Profa. Dra. Flávia Thomaz Verechia Pereira , da Universidade de São Paulo, da Faculdade de Medicina Veterinária e Zootecnia, do Departamento de Cirurgia, pela colaboração no processamento dos tecidos para os exames histopatológicos.

Ao Prof. Dr. José Luiz Guerra, da Universidade de São Paulo, da Faculdade de Medicina Veterinária e Zootecnia, do Departamento de Patologia, pela colaboração nos exames histopatológicos.

À Prefeitura do Campus Administrativo de Pirassununga (PCAPS), em especial ao abtedouro e ao setor de gado de corte. 


\title{
In vitro control of prostaglandin F2a synthesis in bovine endometrium
}

\begin{abstract}
Synthesis of endometrial prostaglandin F2a (PGF2a) results from a complex cascade of highly coordinated intracellular events. Production of PGF2a can be stimulated by mellitin and 12,13 phorbol dibutyrate $(\mathrm{PDBu})$. Objective of the present study was to verify whether basal or stimulated synthesis of PGF2a was dependent on de novo protein synthesis. In Experiment 1, endometrial explants from cyclic, nonlactating cross-bred beef cows $(\mathrm{n}=2)$ on day 15 of the estrous cycle were incubated in quadruplicate in culture KHB culture medium or medium supplemented with $10^{-6} \mathrm{M} \mathrm{PDBu}, 10^{-6} \mathrm{M}$ melittin or $10^{-5} \mathrm{M}$ melittin. Medium samples were collected immediately and 60 minutes after treatment administration and concentration of PGF2a in medium were measured by radioimmunoassay. Concentrations of PGF2a in medium were higher $(\mathrm{P}<0.06)$ after treatment with $\mathrm{PDBu}$ in comparison with control and melittin. In Experiment 2, endometrial explants from cyclic, non-lactating cross-bred beef cows $(n=4)$ on day 17 of the estrous cycle were incubated in quadruplicate in medium supplemented with $0,50,100$ or $200 \mathrm{mg}$ cyclohexamide (CHX) and 0 or $100 \mathrm{ng} / \mathrm{ml} \mathrm{PDBu}$, in a 4 × 2 factorial arrangement. Medium samples were collected immediately and 60 minutes after administration of treatments and concentrations of PGF2a measured by radioimmunoassay. Endometrial integrity was was evaluated by histology. Treatment with PDBu stimulated production of PGF2a $(\mathrm{P}<0.01)$, regardless of concentration of CHX used. The CHX did not affect production of PGF2a, either in the presence or absence of $\mathrm{PDBu}$. Histological integrity of explants was preserved. It was concluded that both basal and PDBu-stimulated PGF2a production are not dependent on new protein sythesis.
\end{abstract}

\section{Referências}

$1 \mathrm{Kim}$, J. J.; Fortier, M. A. A cell type specificity and protein kinase $C$ dependency on the stimulation of prostaglandin E2 and prostaglandin F2a production by oxytocin and platelet-activating factor in bovine endometrial cells. Journal of Reproduction and Fertility, v. 103, p. 239-247, 1995.

2 Asselin, E.; et al. Influence of sex steroids on the production of prostaglandin F2a and E2 and response to oxytocin in cultured epithelial and stromal cells of the bovine endometrium. Biology of Reproduction, $v$. 54, p. 371-379, 1996.

3 Skarzynski, D.; Bogacki, M.; Kotwica, J. Involvement of ovarian steroids in basal and oxytocin-stimulated prostaglandin F2a secretion from bovine endometrium in vitro. Therigenology, v. 52, p. 385-397, 1999.

4 McCracken, J. A.; Glew, M. E.; Scaramuzzi, R. J. Corpus luteum regression induced by prostagland in F2-alpha. Journal of Clinical Endocrinology and Metabolism, v. 30, n. 4, p. 544-546, 1970.

5 McCracken, J. A.; Carlson, J. C.; Glew, M. E.
Keywords:

Cattle.

Prostaglandin. Estradiol. Endometrium. Luteolysis.
Prostaglandin F2 identified as a luteolytic hormone in sheep. Nature: New Biology, v. 238, n. 83, p. 129134, 1972.

6 McCracken, J. A.; Barcikowski, B.; Carlson, J. C. The physiological role of prostaglandin F2alpha in corpus luteum regression. Advanced Bioscience, v. 9, p. 599624, 1973.

7 Meidan, R.; Milvae, R. A.; Weiss, S. Intraovarian regulation of luteolysis. Journal of Reproduction and Fertility Suppl, v. 54, p. 217-228, 1999.

8 Carambula, S. F., Maitikainem, T., Aitikainnem, T. Caspase-3 is a pivotal mediator of apoptosis during regression of ovarian corpus luteum. Endocrinology, v. 143, n. 4, p. 1495-1501, 2002.

9 Davis, S. R.; Collier, R. J.; McNamara, J. P. Effects of thyroxine and growth hormone treatment of dairy cows on mammary uptake of glucose, oxygen and other milk fat precursors. Journal of Animal Science, v. 66, p. 8089, 1988.

10 Chen, G. T. C.; Getsios, S.; MacCalman. 17-betaestradiol potentiates the stimulatory effects of progesterone on cadherin-11 expression in cultured 
human endometrial cells. Endocrinology, v. 139, p. 3512-3519, 1998.

11 Nancarrow, C. D.; Buckmaster, J.; Chameley, W. Hormonal changes around oestrus in the cow. Journal of Reproduction and Fertility, v. 32, n. 2, p. 320-321, 1973.

12 Thorburn, G. D.; et al. Prostaglandin F and progesterone concentrations in the utero-ovarian venous plasma of the ewe during the oestrous cycle and early pregnancy. Journal of Reproduction and Fertility. p. 151, 1973. Supplement 18.

13 Peterson, A. J.; et al. Hormonal changes around bovine luteolysis. Prostaglandins. v. 10, p. 551, 1975.

14 Kindahl, H.; et al. The release of prostaglandin F2a as reflected by 15-keto-13, 14-dihydroprostaglandin F2a in the peripheral circulation during normal luteolysis in heifers. Prostaglandins, v. 11, p. 871, 1996a.

15 Fredrickson, G.; Kindahl, H.; Edqvist, L. E. 11 Ketotetranor PGF metebolites, a suitable indicator for measuring prostaglandin release during the normal oestruscycle and early pregnancy in the goat. Animal Reproduction Science, v. 7, p. 537, 1984.

16 Burns, P. D.; et al. Cellular mechanisms by which oxytocin stimulates uterine PGF2a synthesis in bovine endometrium: roles of phospholipases $\mathrm{C}$ and $\mathrm{A} 2$. Domestic Animal Endocrinology, v. 14, n. 3, p. 181 191, 1997.

17 Alberts, B; et al. Biologia molecular da célula. 3. ed. Porto Alegre: Artes Médicas, 1997. 1294 p.

18 Clark, J. D.; et al. A novel arachidoinic acid-selective cytosolic PLA2 contain a Ca $(2+)$ - dependent transloation domains witho homology to PKC and GAP. Cell, v. 65, p. 1043-1051, 1991.

19 Gijon, M. A.; Leslie, C. C. Regulation of arachidonic acid release and cytosolic phospholipase A2 activation. Journal of Leukocites Biology, v. 65, p. 330-336, 1999.

20 Silvia, W. J.; et al. Hormonal regulation of uterine secretion of PGF2a durin luteolysis in ruminants. Biology of Reproduction, v. 45, p. 655-663, 1991.

21 Diaz, F. J.; et al. Regulation of progesterone and prostaglandin F2a production in the CL. Molecular and Cellular Endocrinology, v.191, p. 65-8, 2002.

22 Lee, J. S.; Silvia, W. J. Cellular mechanisms mediating the stimulation of ovine endometrial secretion of prostaglandin F2a in response to oxytocin: role of phospholipase A2. Journal of Endocrinology, v. 141, p. 491, 1994.

23 SAS. SAS/STAT TM user's guide ( Release 6.03 ). Cary, NC: SAS Institute Inc.; 1988.

24 Silvia, W. J.; et al. Cellular mechanisms mediating the stimulation of ovine endometrial secretion of prostaglandin $\mathrm{F} 2 \mathrm{a}$ in response to oxytocin: role of phospholipase $C$ and diacylglicerol. Journal of
Endocrinology, v. 141, p. 481, 1994.

25 Silvia, W. J.; Homanics, G. E. Role of phospholipase $\mathrm{C}$ in mediating oxytocin-induced release of prostaglandin F2a from ovine endometrial tissue. Prostaglandins, v. 35, p. 535, 1988.

26 Tysseling, K. A.; et al. Mechanisms regulating prostaglandin F2asecretion from the bovine endometrim. Journal of Dairy Science. v. 81, p. 382, 1998.

27 Danet-Desnoyers, G.; et al. Regulation of endometrial prostaglandin synthesis during early pregnancy in cattle: effects of phospholipase and calcium in vitro. Prostaglandins, v. 50, p. 313-330, 1995.

28 Peltier, M. R.; Wilcox, C. J.; Sharp, D. C. Technical note: Aplication of box-cox data transformation to animal science experiments. Journal of Animal Science, v. 76, p. 846-849, 1998.

29 Arnold, D. R.; et al. Intracellular regulation of endometrial PGF2a production in dairy cows during early pregnancy and following treatment with recombinant interferon t. Domestic Animal Endocrinology, v. 18, p. 199-216, 2000.

30 Binelli, M.; et al. Characterization of an in vitro perifusion system to study endometrial function in cattle. Biology of Reproduction, v. 66, p. 323, 2002. Supplement. 1.

31 Binelli, M.; Bertan, C. M.; Cunha, P. M. Estradiol$17 \mathrm{~b}$ potentiates calcium-stimulated prostaglandin F2a release from bovine endometrium. In: Annual Meeting, 37, Vancouver, Canada, 2004. Biology of Reproduction, 2004, v. 70 (Suppl. 1), no prelo.

32 Mattson, M. P.; Furukawa, K. Anti-apoptotic actions of cyclohexamide: blockade of programmed cell death or induction of programmed cell life? Apoptosis, v. 2, p. 257-264, 1777. 